\title{
Ivabradine in acute heart failure: Effects on heart rate and hemodynamic parameters in a randomized and controlled swine trial
}

Marina Pascual Izco ${ }^{1}$, Rafael Ramírez-Carracedo², Ignacio Hernández Navarro², Álvaro Osorio Ruiz ${ }^{3}$, Borja Castejón Navarro ${ }^{4}$, Irene Cuadrado Berrocal ${ }^{5}$, Carlota Largo Aramburu ${ }^{6}$, Gonzalo Luis Alonso Salinas ${ }^{1,7}$, Javier Díez ${ }^{2}$, Marta Saura Redondo ${ }^{5}$, José Luis Zamorano ${ }^{1,7}$, Carlos Zaragoza ${ }^{2}$, Marcelo Sanmartin ${ }^{1,7}$

${ }^{1}$ Cardiology Department, Ramón y Cajal University Hospital (IRYCIS),

University of Alcalá de Henares, Madrid, Spain

${ }^{2}$ Cardiology Department, Cardiovascular Research Unit. Francisco de Vitoria University

- Ramón y Cajal University Hospital (IRYCIS), Madrid, Spain

${ }^{3}$ Vascular Surgery Department, Ramón y Cajal University Hospital (IRYCIS), Madrid, Spain

${ }^{4}$ Cardiovascular Research Unit, Ramón y Cajal University Hospital (IRYCIS), Madrid, Spain ${ }^{5}$ Physiology Unit, Systems Biology Department, University of Alcalá de Henares, Madrid, Spain

${ }^{6}$ Experimental Surgery Department, Hospital Universitario La Paz, Madrid, Spain

${ }^{7}$ CIBERCV, Ramón y Cajal University Hospital (IRYCIS), Madrid, Spain

Abstract

Background: Acute heart failure patients could benefit from heart rate reduction, as myocardial consumption and oxidative stress are related to tachycardia. Ivabradine could have a clinical role attenuating catecholamine-induced tachycardia. The aim of this study was to evaluate hemodynamic effects of ivabradine in a swine model of acute heart failure.

Methods: Myocardial infarction was induced by 45 min left anterior descending artery balloon occlusion in 18 anesthetized pigs. An infusion of dobutamine and noradrenaline was maintained aiming to preserve adequate hemodynamic support, accompanied by fluid administration to obtain a pulmonary wedged pressure $\geq 18 \mathrm{mmHg}$. After reperfusion, rhythm and hemodynamic stabilization, the animals were randomized to $0.3 \mathrm{mg} / \mathrm{kg}$ ivabradine intravenously $(n=9)$ or placebo $(n=9)$. Hemodynamic parameters were observed over a 60 min period.

Results: Ivabradine was associated with a significant reduction in heart rate $(88.4 \pm 12.0 \mathrm{bpm} v \mathrm{vs}$. $122.7 \pm 17.3 \mathrm{bpm}$ after $15 \mathrm{~min}$ of ivabradine/placebo infusion, $p<0.01)$ and an increase in stroke volume (68.8 $\pm 13.7 \mathrm{~mL}$ vs. $52.4 \pm 11.5 \mathrm{~mL}$ after $15 \mathrm{~min}, p=0.01)$. There were no significant differences in systemic or pulmonary arterial pressure, or significant changes in pulmonary capillary pressure. However, after 15 min, cardiac output was significantly reduced with ivabradine $(-5.2 \%$ vs. $+15.0 \%$ variation in ivabradine/placebo group, $p=0.03)$, and central venous pressure increased $(+4.2 \%$ vs. $-19.7 \%$ variation, $p<0.01$.

Conclusions: Ivabradine reduces heart rate and increases stroke volume without modifying systemic or left filling pressures in a swine model of acute heart failure. However, an excessive heart rate reduction could lead to a decrease in cardiac output and an increase in right filling pressures. Future studies with specific heart rate targets are needed. (Cardiol J 2020; 27, 1: 62-71)

Key words: acute heart failure, heart rate, ivabradine, swine model, porcine model

Address for correspondence: Dr. Marcelo Sanmartin Fernandez, Cardiology Department, Ramón y Cajal University Hospital, Ctra. de Colmenar Viejo, km. 9100, 28034 Madrid, Spain, tel: 0034-913368097, e-mail: msanfer@me.com 


\section{Introduction}

Acute heart failure (AHF) continues to be associated with poor prognosis and high in-hospital mortality [1]. Inotropic drugs are the cornerstone in management of hypotensive (cold) AHF patients, promoting a dose-dependent increase in cardiac output (CO) and reduction of left ventricle (LV) filling pressures. The downside to these effects is an increase in heart rate (HR) with its corresponding increase in myocardial oxygen consumption [2-5]. European Society of Cardiology guidelines recommend a limited use of inotropic agents in the management of AHF [1], recognizing sinus tachycardia as possibly deleterious, as it may compromise ventricular filling efficiency by shortening diastolic duration [6]. Accordingly, reduction of HR could be an important energy-saving maneuver in AHF.

Ivabradine is a specific blocker of the If "funny" currently supported by hyperpolarization-activated cyclic nucleotide-gated channels, regulating sinoatrial node activity. Its pharmacological effects are deemed to be almost exclusively on the sinus node reducing HR without affecting myocardial contractility or vascular tone. Ivabradine has been shown to reduce re-hospitalization and mortality rates in patients with chronic $\mathrm{HF}$ and $\leq 35 \%$ left ventricular ejection fraction (LVEF) $[1,7,8]$, but it has not been adequately tested in AHF. It has been postulated that ivabradine could have a role attenuating catecholamine-induced tachycardia [5, $6]$. Few case series and reports suggest its safety and potential benefit, improving surrogate endpoints in AHF and cardiogenic shock (CS) [9-12]. However, the effects of rapid reduction of $\mathrm{HR}$ on $\mathrm{CO}$ or heart filling pressures in this context are not clearly understood and a comprehensive analysis of hemodynamic of ivabradine effects in this setting is warranted.

The main objective of this study was to evaluate the impact of ivabradine on hemodynamic parameters in a swine model of AHF.

\section{Methods}

\section{Ethical approval}

All procedures were performed in the Experimental Surgery Department of the Hospital Universitario La Paz (Madrid, Spain). Protocol was followed and approved by the Animal Welfare Ethics Committee. The investigation conforms to Guide for the Care and Use of laboratory Animals, published by the US National Institutes of Health
(NIH Publication No. 85-23, revised 1985) and complied with the EU Directive on experimental animals (63/2010 EU) and related Spanish legislation (RD 53/2013). PROEX 365-15.

\section{Surgery and instrumentation}

Eighteen Large White female pigs $(37.6 \pm 5.1 \mathrm{~kg})$ were used in the study. The animals were initially treated with intramuscular ketamine $(10 \mathrm{mg} / \mathrm{kg}$, Pfizer) and midazolam (0.5 mg/kg, Braun). Anesthesia was induced by inhaled isoflurane (Abbvie Spain SLU) and maintained with continuous infusion of propofol $(2 \mathrm{~mL} / \mathrm{kg} / \mathrm{h}$, Fresenius Kabi), fentanyl ( $50 \mu \mathrm{g} / \mathrm{kg} / \mathrm{h}$, Kern Pharma) and diazepam $(10 \mu \mathrm{g} / \mathrm{kg} / \mathrm{h}$, Roche). Animals were intubated and ventilated with $60 \%$ oxygen saturation. A central venous catheter (Swan-Ganz oximetry thermodilution catheter, Edwards Lifesciences) was placed through the right internal jugular vein to the pulmonary artery for measurement of central venous pressure (CVP), CO, pulmonary capillary wedge pressure (PCWP), pulmonary artery pressure (PAP) and mixed venous oxygen saturation $\left(\mathrm{SvO}_{2}\right)$. Systemic arterial pressure (SAP) was measured in the ascending aorta through the guiding catheter used to catheterize the left coronary artery tree. The animals received 5000 IU of heparin and amiodarone ( $53 \mu \mathrm{g} / \mathrm{kg} / \mathrm{min}$, Sanofi Aventis) to avoid blood clotting of catheters and malignant cardiac arrhythmias, respectively. Animals were stabilized for $15 \mathrm{~min}$ before baseline measurements (T0).

\section{Experimental protocol}

The experimental protocol is outlined in Figure 1. After baseline measurements, including echocardiogram and hemodynamic assessment, acute anterior myocardial infarction was induced by midleft anterior descending artery (LAD) occlusion for $45 \mathrm{~min}$ using a JL $36 \mathrm{~F}$ catheter and 3.0 or $3.5 \mathrm{~mm}$ conventional angioplasty balloons. Hemodynamic measurements were continuously monitored and recorded at 15, 30 and 45 min during ischemia (T15, T30, T45). Noradrenaline $(0.4-0.8 \mu \mathrm{g} / \mathrm{kg} / \mathrm{min}$, Braun), dobutamine (2.9-6.2 $\mu \mathrm{g} / \mathrm{kg} / \mathrm{min}$, Hospira Productos farmacéuticos y Hospitalarios SL) and physiological saline (1000-2000cc, Grifols) were administered after the first $15 \mathrm{~min}$ of occlusion to maintain adequate systemic perfusion and titrated to obtain a $\mathrm{HR} \geq 90 \mathrm{bpm}$ and PCWP $>18 \mathrm{mmHg}$. After 45 min of coronary occlusion, the angioplasty balloon was deflated and at least 15 min of electrical and hemodynamic stabilization time was allowed before infusion of study drug or placebo. Hemodynamic measurements were recorded (T60) and 


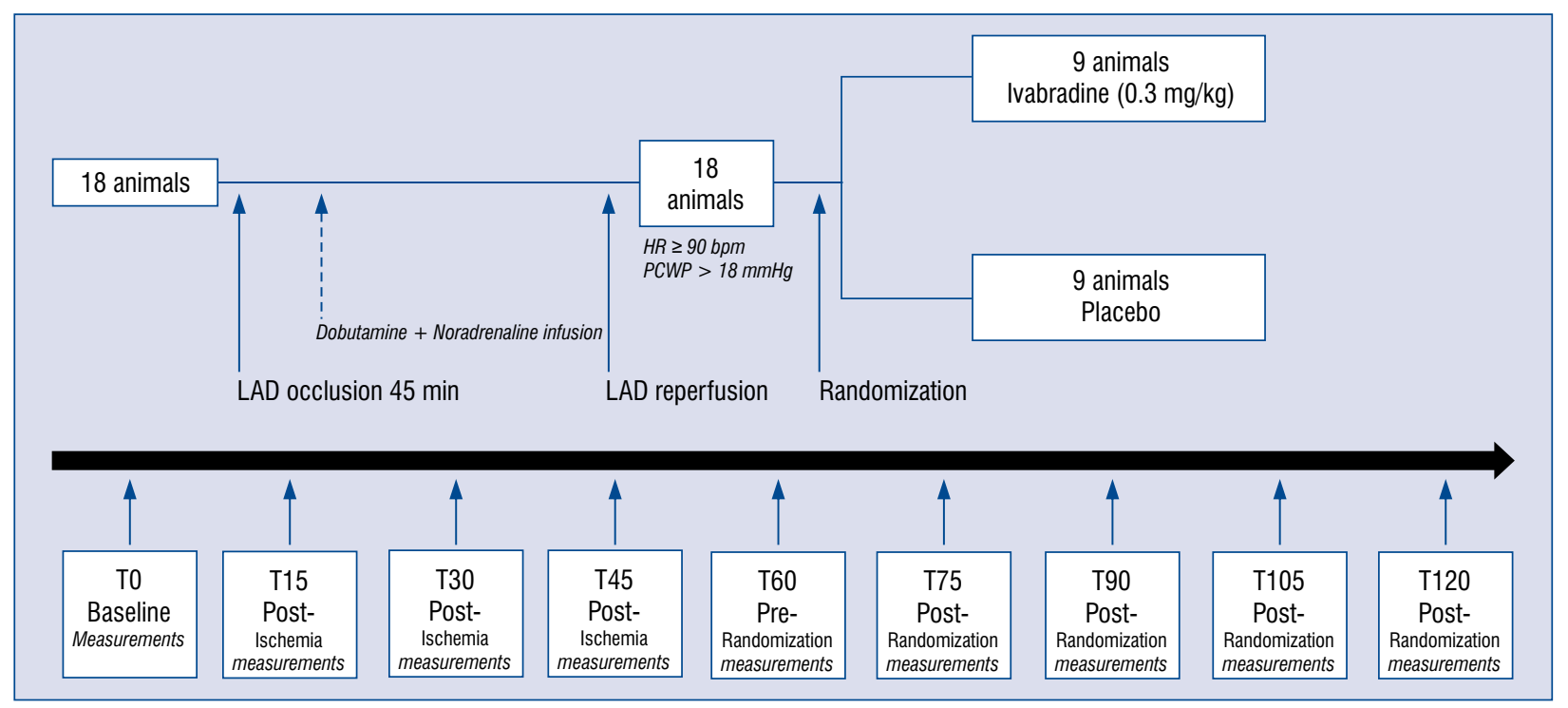

Figure 1. Outline of the experimental protocol. After baseline assessment of hemodynamics parameters, animals underwent left anterior descending artery (LAD) occlusion. Hemodynamic measurements were recorded at 15, 30 and $45 \mathrm{~min}$ of ischemia. After LAD reperfusion, pigs were allowed to stabilize for another 15 min before recording the pre-randomization measurements. Animals were then randomized to receive ivabradine $(0.3 \mathrm{mg} / \mathrm{kg}) \mathrm{of}$ either placebo. Hemodynamic measurements were repeated 15-30-45 and 60 min after ivabradine/placebo administration; HR — heart rate; PCWP — pulmonary capillary wedge pressure.

animals were then randomized to a control group $(\mathrm{n}=9)$ or to receive ivabradine $(\mathrm{n}=9)$. According to Servier laboratories instructions, ivabradine powder was diluted in distilled water in a proportion of at least $12 \mathrm{mg} / \mathrm{mL}$ and administered intravenously in slow bolus at a dose of $0.3 \mathrm{mg} / \mathrm{kg}$. This dose was chosen based on evidence available in the literature on the effect of intravenously ivabradine in porcine and human models, with doses between $0.1 \mathrm{mg} / \mathrm{kg}$ and $0.6 \mathrm{mg} / \mathrm{kg}$ [13-16]. The control group received an equivalent volume of physiological saline. Hemodynamic measurements were repeated 15-30-45 and $60 \mathrm{~min}$ after ivabradine/placebo administration (T75, T90, T105, T120).

\section{Statistical analysis}

All data were analyzed with a statistical software package (Stata 13.0 statistical software (Stata Corporation, College Station, TX)). All values are given as mean \pm standard deviation. A two-sided $\mathrm{p}<0.05$ was considered significant. Saphiro-Wilk test was used to assess variable distribution.

Statistical analysis was performed in two phases. Firstly, individual variables were assessed before and after coronary occlusion in the same animal. The Student t-test for paired data was used to analyze variables of normal distribution, and Wilcoxon test was used in non-normal distribution variables. Secondly, hemodynamic effects of ivabradine were compared with placebo. The Student t-test for unpaired data was used in variables of normal distribution, and Mann-Whitney U test was used in variables of non-normal distribution.

\section{Results}

\section{Effects of coronary occlusion on hemodynamic parameters}

The accumulated ischemia time was $45.3 \pm$ $\pm 1.2 \mathrm{~min}$. Ventricular fibrillation necessitating external defibrillation occurred on average 1.9 times per animal. The induction of ischemia led to a significant decline in SAP and CO in the first $15 \mathrm{~min}$ after LAD occlusion (Table 1). Following dobutamine and noradrenaline administration, HR, PCWP, PAP and CVP increased progressively (Table 1).

\section{Effects of ivabradine on \\ hemodynamic parameters}

Hemodynamic variables for ivabradine-treated animals and controls are summarized in Tables 2 and 3 and Figure 2. Table 2 expresses absolute values. Study groups had comparable HR and systemic or pulmonary perfusion and filling pressures at baseline. Ivabradine administration produced a significant reduction in HR (at 15 min: $-21.9 \%$ reduction vs. $2.6 \%$ increase; $\mathrm{p}<0.01$ ). Parallel to this rapid reduction in HR, we observed a sig- 
Table 1. Parameters before left anterior descendent artery (LAD) occlusion; parameters 15,30 and 45 min after LAD occlusion and 15 min after reperfusion.

\begin{tabular}{|c|c|c|c|c|c|}
\hline \multicolumn{6}{|c|}{ Effects of coronary occlusion on hemodynamic parameters } \\
\hline \multirow{2}{*}{$\begin{array}{l}\text { Hemodynamic } \\
\text { parameters } \\
(n=18)\end{array}$} & \multirow[b]{2}{*}{ Basal } & \multicolumn{3}{|c|}{ LAD occlusion } & \multirow{2}{*}{$\begin{array}{c}\text { LAD reperfusion } \\
+D B / N A\end{array}$} \\
\hline & & $\begin{array}{l}\text { LAD } \\
15 \mathrm{~min}\end{array}$ & $\begin{array}{l}\text { LAD } 30 \text { min } \\
+ \text { DB/NA }\end{array}$ & $\begin{array}{l}\text { LAD } 45 \text { min } \\
+ \text { DB/NA }\end{array}$ & \\
\hline SAP $[\mathrm{mmHg}]$ & $95.1 \pm 14.4$ & $87.2 \pm 14.2^{*}$ & $95.3 \pm 24.3$ & $101.2 \pm 12.5$ & $105.2 \pm 12.3^{*}$ \\
\hline MAP [mmHg] & $73.6 \pm 10.2$ & $68.1 \pm 12.5$ & $74.7 \pm 20.4$ & $78.3 \pm 12.5$ & $71.2 \pm 11.4$ \\
\hline $\mathrm{CO}[1 / \mathrm{min}]$ & $5.2 \pm 1.1$ & $4.6 \pm 1.3^{*}$ & $5.0 \pm 1.2$ & $5.6 \pm 1.1$ & $6.10 \pm 1.4^{*}$ \\
\hline $\mathrm{SV}[\mathrm{mL}]$ & $59.9 \pm 12.0$ & $51.6 \pm 15.5^{* *}$ & $50.9 \pm 14.0^{* *}$ & $56.3 \pm 12.8$ & $51.4 \pm 11.6^{* *}$ \\
\hline $\mathrm{HR}$ [bpm] & $88.1 \pm 17.5$ & $90.3 \pm 13.6$ & $98.8 \pm 13.0^{*}$ & $99.9 \pm 12.5^{*}$ & $116.4 \pm 16.5^{* *}$ \\
\hline PCWP [mmHg] & $16.0 \pm 3.0$ & $18.8 \pm 3.0^{* *}$ & $21.1 \pm 3.9 * *$ & $20.9 \pm 3.0 * *$ & $23.4 \pm 3.8^{* *}$ \\
\hline MPAP $[\mathrm{mmHg}]$ & $23.7 \pm 5.2$ & $24.4 \pm 5.7$ & $25.7 \pm 6.6$ & $27.9 \pm 6.0^{* *}$ & $31.4 \pm 6.5^{* *}$ \\
\hline CVP $[\mathrm{mmHg}]$ & $9.9 \pm 2.9$ & $11.4 \pm 2.8^{*}$ & $12.6 \pm 3.1^{* *}$ & $13.5 \pm 3.0^{* *}$ & $13.7 \pm 3.2^{* *}$ \\
\hline $\mathrm{SVO}_{2}[\%]$ & $69.4 \pm 12.6$ & $65.0 \pm 7.0$ & $72.3 \pm 8.9$ & $77.7 \pm 6.8^{*}$ & $76.9 \pm 7.4^{*}$ \\
\hline
\end{tabular}

Values are expressed as mean \pm standard deviation. LAD occlusion led to a significant decline in MAP, CO and SV in the first 15 min after LAD occlusion. Dobutamine and noradrenaline, along with fluids, were administered after 15 min of occlusion to preserve adequate hemodynamics during induction of myocardial infarction. ${ }^{*} \mathrm{p}<0.05$; ${ }^{*} \mathrm{p}<0.01$. $\mathrm{N}=18$ (all animals, both groups); DB - dobutamine; NA - noradrenaline; SAP - systolic arterial pressure; MAP - mean arterial pressure; CO — cardiac output; SV - stroke volume; HR - heart rate; PCWP

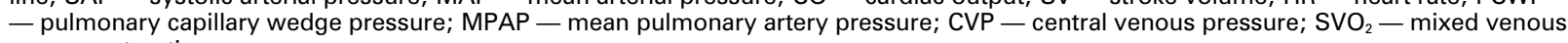
oxygen saturation

nificant $\mathrm{CO}$ reduction in response to ivabradine (at 15 min: $-5.2 \%$ reduction vs. $+15.0 \%$ increase; $\mathrm{p}=0.03)$, that remained stable during the following 45 min (Table 3, Fig. 2). Despite reduction of HR and $\mathrm{CO}$ no significant changes in perfusion or left filling pressures were observed (SAP, PCWP and PAP in Tables 2 and 3 and Fig. 2). After ivabradine bolus there was a corresponding increase in stroke volume (SV), compared to placebo values $(+21.5 \%$ vs. $+13.6 \%$ increase, ivabradine and placebo respectively; $\mathrm{p}=0.34$ ), which tended to stabilize during the $60 \mathrm{~min}$ observation period (Table 2 , Fig. 2). Finally, a significant reduction in right filling pressure was observed (CVP) in the control group, and not observed in the ivabradine-assigned group $(+4.2 \%$ increase vs. $-19.7 \%$ reduction after $15 \mathrm{~min}$ ivabradine/placebo administration, $\mathrm{p}<0.01$ ).

\section{Discussion}

This is an open-label randomized study evaluating the hemodynamic effects of ivabradine in an experimental model of AHF based on an acute myocardial infarction-reperfusion injury. LAD occlusion led to a significant decline in mean arterial pressure (MAP) and CO similar to other studies with the same characteristics [17]. The rapid reduction of $\mathrm{HR}$ induced by an intravenous bolus of ivabradine produced a reduction in $\mathrm{CO}$ with a corresponding increase in SV, with no changes in systemic or pulmonary arterial pressures. There were no acute changes in left filling pressures, while there was a spontaneous decrease in right filling pressures in control animals, not observed in the ivabradine group. An observed reduction in $\mathrm{CO}$ was not progressive and both perfusion and heart filling pressures tended to remain stable after the first hour of drug administration.

Tachycardia is a common and physiological response to vasodilation or reduction in effective perfusion pressures but can result in higher myocardial oxygen consumption and lower coronary filling time. Higher HR has been shown to predict a worse prognosis in both AHF and chronic decompensated HF $[18,19]$. After demonstration of reduction of $\mathrm{HR}$ with ivabradine in chronic $\mathrm{HF}$ [7, 8], several studies have suggested a possible role of rapid lowering HR during hospitalization in $\mathrm{AHF}$ patients or CS [9-12, 20-23]. However, few data are available on the hemodynamics of rapidly reducing HR in AHF. Gallet et al. [9] evaluated the effects of combining dobutamine and ivabradine ( $5 \mathrm{mg}$ bid) in 9 patients with refractory CS. Ivabradine not only reduced $\mathrm{HR}$, but also improved SAP, CO and N-terminal pro-B-type natriuretic peptide (NT-proBNP) values. Cavusoglu et al. [22] randomized 58 patients with $\mathrm{AHF}$ requiring inotropic support to receive ivabradine $(n=29)$ or placebo $(n=29)$ in addition to dobutamine. In the control group, mean HR gradually and significantly increased 


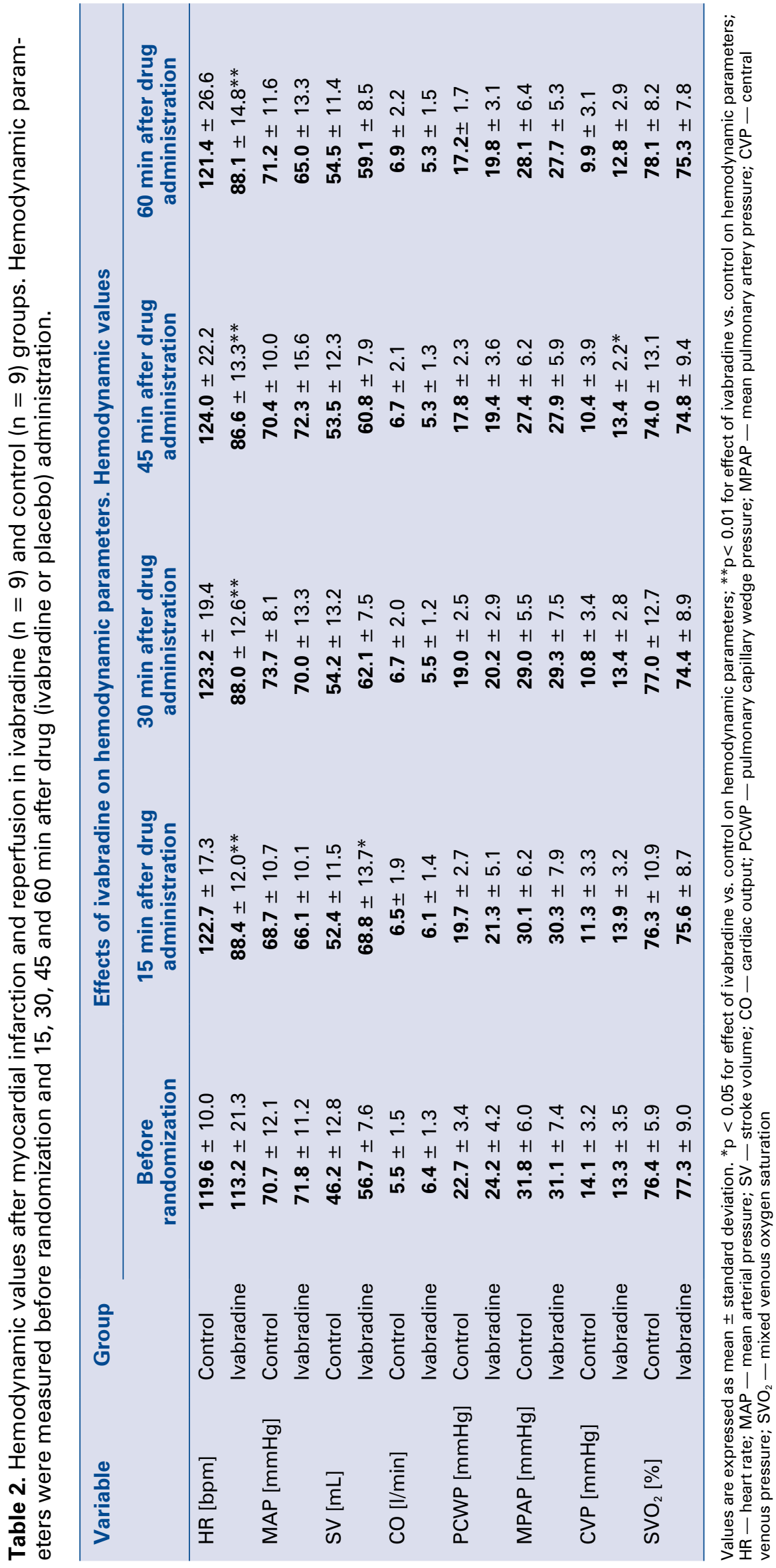




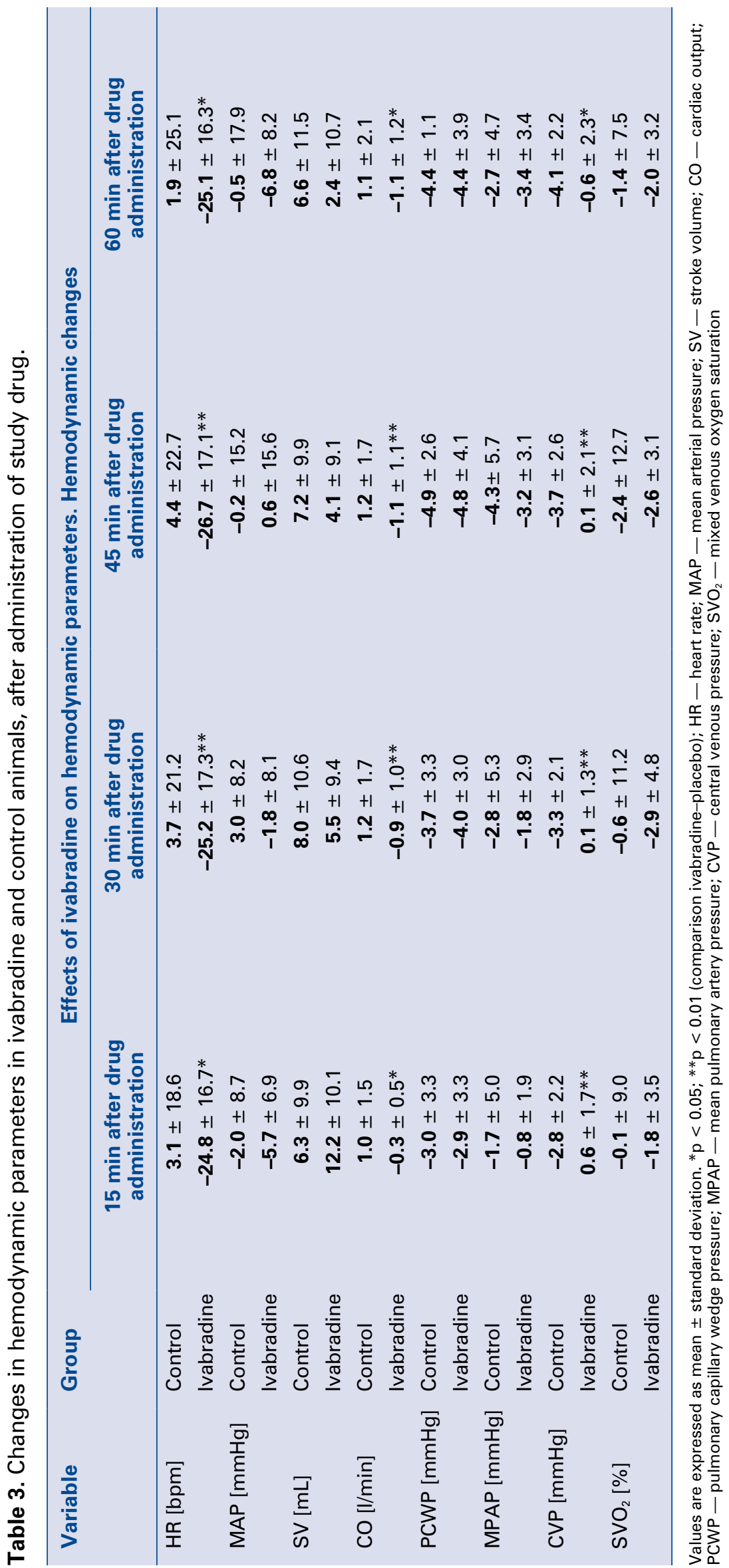


Cardiology Journal 2020, Vol. 27, No. 1
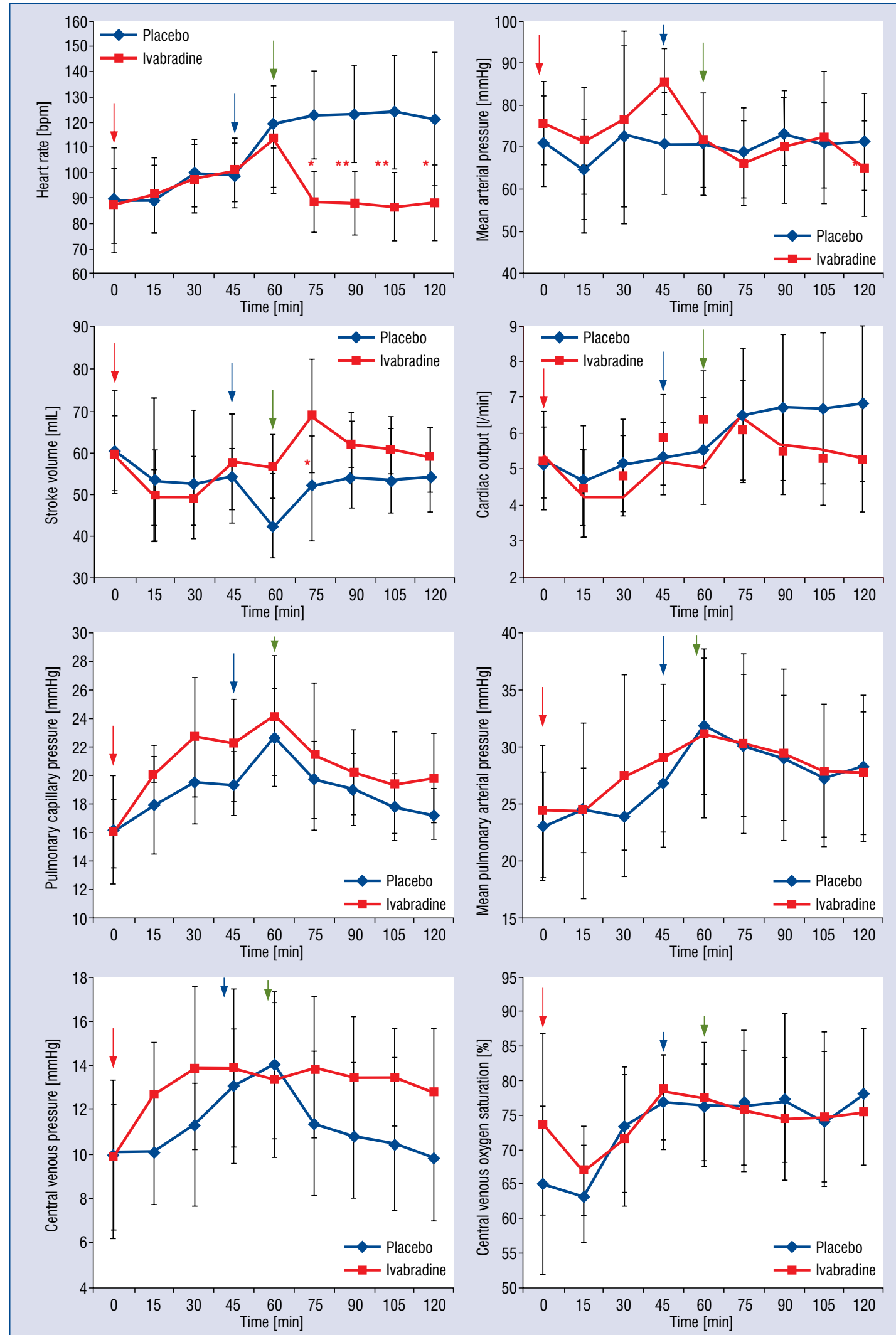

$\rightarrow$ Left anterior descending artery occlusion

$\rightarrow$ Reperfusion

$\rightarrow$ Drug administration (ivabradine or placebo)

Figure 2. Ivabradine and placebo hemodynamic parameters variations; ${ }^{*} p<0.05$ for effect of ivabradine vs. placebo on hemodynamic parameters; ${ }^{* *} \mathrm{p}<0.01$ for effect of ivabradine vs. placebo on hemodynamic parameters; $\mathrm{N}=9$ — ivabradine group; $\mathrm{N}=9$ — placebo group. 
at each step of dobutamine infusion, whereas no significant increase in HR was observed in the ivabradine group. However, important hemodynamic parameters, such as PCWP, SAP or CO were not recorded in these studies. In a study by Barillá et al [23], a total of 58 patients with CS as a complication of acute elevation myocardial infarction and HR $>75 \mathrm{bpm}$ were randomized to standard treatment or to standard treatment plus ivabradine. HR was significantly reduced in ivabradine group, without modifying SAP. In-hospital mortality was doubled in the standard group in comparison with the standard plus ivabradine group, but the difference was not statistically significant. Nevertheless, invasive hemodynamic parameters such as $\mathrm{CO}$ or SV were not measured in this study. The MODIFY trial [24] enrolled 70 patients with multiple organ dysfunction syndrome (MODS) and HR $>90 \mathrm{bpm}$. Patients were randomized to receive standard treatment plus ivabradine or placebo. The HR reduction from enteral administration of ivabradine was not associated with an improvement in hemodynamic values or disease severity among critically ill patients with MODS. However, this study included patients with MODS but not necessarily with CS or AHF. In fact, coronary etiology was identified in only 17 patients; but important information as LVEF or filling pressure values was not registered. On the other hand, patients enrolled in the MODIFY trial received mainly vasopressor drugs instead of inotropic drugs. The present study focuses on the role of ivabradine in tachycardia induced by inotropic drugs (mainly dobutamine) in patients with AHF and depressed LVEF. Target HR may not be the same for patients with preserved or reduced cardiac inotropism, but the effect of ivabradine on hemodynamic parameters in this kind of patient have not been studied in any human clinical trial yet.

In one experimental swine model, Bakkehaug et al. [25] presented an ischemia-reperfusion protocol in 12 pigs after intermittent ligation of the left coronary arteries. Dobutamine infusion in post-ischemic heart increased $\mathrm{CO}$ by increasing significantly HR from 102 to $131 \mathrm{bpm}(\mathrm{p}<0.05)$. The analysis demonstrated that adding ivabradine to dobutamine reported a reduction of HR to baseline values $(100 \mathrm{bpm})$ without any effect on $\mathrm{CO}$ or MAP, with a significant increase in SV. However, in this study the hemodynamic effects of ivabradine were not determined in different groups, but the same animal was compared with itself before and after ivabradine administration. Therefore, it is impossible to overrule a "self-recovery" effect after releasing left coronary occlusion.

In the present study, ivabradine significantly reduced HR with no effect on SAP or PCWP. In contrast to previous observations and despite a rapid increase in SV, a decrease in $\mathrm{CO}$ values was observed when compared to control animals. However, the following limitations should be taken into consideration. First of all, the observation period (60 $\mathrm{min}$ ) could be too short to detect a possible "catch-up" effect on CO values or stabilization of right or left filling pressures. Secondly, the experimental model in this study was unable to produce a sustained reduction in $\mathrm{CO}$ as in pump failure due to acute myocardial infarction and actually several animals showed a high-CO state induced by catecholamine infusion. And finally, this is a modest sample. Thus, caution is suggested in extrapolating this observed effect on CO to "clinical CS". Despite these important limitations, it is believed herein that rapid and excessive HR reduction as produced by this model might be inappropriate in a clinical scenario. Accordingly, it may be postulated that there could be a specific ideal HR target, adjusted to individual clinical scenarios, and that an excessive attenuation of tachycardia response to endogenous or therapeutic catecholamines could be deleterious, leading to increased filling pressures and reduction of $\mathrm{CO}$.

Another unique finding of in the present results was higher CVP values in the ivabradine group compared with the placebo group, with no clear effects on PCWP. It is possible that preserving an adequate $\mathrm{HR}$ in the ischemia-reperfusion model could be more important to right-ventricle filling pressures that left-ventricle mechanics. These data should be confirmed in robust future studies with longer observation times and, ideally, clinical endpoints.

This study has some limitations that should be considered. First, anesthetic drugs could exert a vasodilatory effect not present in clinical models of AHF, although the same anesthetics were used in the ivabradine and control group. Second, dobutamine and noradrenaline were administered to induce tachycardia and maintain adequate systemic perfusion. Cathecolamine infusion produced a high-output state in some experimental animals could have had an important interaction with the hemodynamic effects of the study drug. Finally, operators were blinded to the product administered after the occlusion phase (ivabradine or saline infusion). However, masking the chronotropic effects 
of ivabradine was not possible, reducing the impact of a blind design.

\section{Conclusions}

In conclusion, in a swine model of myocardial ischemia-reperfusion injury and AHF, ivabradine effectively attenuates catecholamine-induced tachycardia and acutely increases SV and diastolic filling time without affecting systemic or left-heart filling pressures. However, in this experimental model, an excessive HR reduction produced lower $\mathrm{CO}$ values. Future studies with more specific HR targets are needed to evaluate possible benefits of ivabradine in this context.

\section{Acknowledgements}

We are thankful for the skillful technical assistance from the Experimental Surgery Department personnel of the Hospital Universitario La $\mathrm{Paz}$ (Madrid, Spain).

Fundings: This work was supported by a basic research grant from the Spanish Society of Cardiology and Carlos III Health Institute, being part of the ES-FISH research project (PI16/01417).

Conflict of interest: None declared. Servier laboratories generously donated the ivabradine powder used in this study.

\section{References}

1. Ponikowski P, Voors AA, Anker SD, et al. 2016 ESC Guidelines for the diagnosis and treatment of acute and chronic heart failure: The Task Force for the diagnosis and treatment of acute and chronic heart failure of the European Society of Cardiology (ESC). Developed with the special contribution of the Heart Failure Association (HFA) of the ESC. Eur J Heart Fail. 2016; 18(8): 891-975, doi: 10.1002/ejhf.592, indexed in Pubmed: 27207191.

2. Hastillo A, Taylor DO, Hess ML. Secific ositiv inotroic agents. In: Messerli FH,ed. Cardiovascular drug theray. 2nd Ed. W G Saunders Company; Philadelphia. 1996: 1151-1161.

3. Tisdale JE, Patel R, Webb CR, et al. Electrophysiologic and proarrhythmic effects of intravenous inotropic agents. Prog Cardiovasc Dis. 1995; 38(2): 167-180, indexed in Pubmed: 7568905.

4. Vasquez A, Kern KB, Hilwig RW, et al. Optimal dosing of dobutamine for treating post-resuscitation left ventricular dysfunction. Resuscitation. 2004; 61(2): 199-207, doi: 10.1016/j.resuscitation.2004.01.002, indexed in Pubmed: 15135197.

5. Roubille F, Lattuca B, Busseuil D, et al. Is ivabradine suitable to control undesirable tachycardia induced by dobutamine in cardiogenic shock treatment? Med Hypotheses. 2013; 81(2): 202-206, doi: 10.1016/j.mehy.2013.05.002, indexed in Pubmed: 23719030.

6. Akodad M, Lim P, Roubille F. Does ivabradine balance dobutamine effects in cardiogenic shock? A promising new strategy.
Acta Physiol (Oxf). 2016; 218(2): 73-77, doi: 10.1111/apha.12733, indexed in Pubmed: 27291979.

7. Fox K, Ford I, Steg P, et al. Ivabradine for patients with stable coronary artery disease and left-ventricular systolic dysfunction (BEAUTIFUL): a randomised, double-blind, placebo-controlled trial. Lancet. 2008; 372(9641): 807-816, doi: 10.1016/s01406736(08)61170-8.

8. Swedberg K, Komajda M, Böhm M, et al. Ivabradine and outcomes in chronic heart failure (SHIFT): a randomised placebocontrolled study. Lancet. 2010; 376(9744): 875-885, doi: 10.1016/ s0140-6736(10)61198-1.

9. Gallet R, Ternacle J, Damy T, et al. Hemodynamic effects of Ivabradine in addition to dobutamine in patients with severe systolic dysfunction. Int J Cardiol. 2014; 176(2): 450-455, doi: 10.1016/j.ijcard.2014.07.093, indexed in Pubmed: 25129291.

10. Bonadei I, Sciatti E, Vizzardi E, et al. Ivabradine during cardiogenic shock: A clinical case and review of the literature. Heart Lung J Crit Care. 2015; 44(1): 57-58, doi: 10.1016/j. hrtlng.2014.08.003.

11. Hidalgo FJ, Anguita M, Castillo JC, et al. Effect of early treatment with ivabradine combined with beta-blockers versus betablockers alone in patients hospitalised with heart failure and reduced left ventricular ejection fraction (ETHIC-AHF): A randomised study. Int J Cardiol. 2016; 217: 7-11, doi: 10.1016/j. ijcard.2016.04.136, indexed in Pubmed: 27167103.

12. Pascual Izco M, Alonso Salinas GL, Sanmartín Fernández M, et al. Clinical Experience with Ivabradine in Acute Heart Failure. Cardiology. 2016; 134(3): 372-374, doi: 10.1159/000444845, indexed in Pubmed: 27100325.

13. Heusch G, Skyschally A, Gres P, et al. Improvement of regional myocardial blood flow and function and reduction of infarct size with ivabradine: protection beyond heart rate reduction. Eur Heart J. 2008; 29(18): 2265-2275, doi: 10.1093/eurheartj/ehn337, indexed in Pubmed: 18621770.

14. Verrier RL, Silva AFG, Bonatti R, et al. Combined actions of ivabradine and ranolazine reduce ventricular rate during atrial fibrillation. J Cardiovasc Electrophysiol. 2015; 26(3): 329-335, doi: 10.1111/jce.12569, indexed in Pubmed: 25346368.

15. Verrier RL, Bonatti R, Silva AFG, et al. If inhibition in the atrioventricular node by ivabradine causes rate-dependent slowing of conduction and reduces ventricular rate during atrial fibrillation. Heart Rhythm. 2014; 11(12): 2288-2296, doi: 10.1016/j. hrthm.2014.08.007, indexed in Pubmed: 25111327.

16. Camm AJ, Lau CP. Electrophysiological effects of a single intravenous administration of ivabradine (S 16257) in adult patients with normal baseline electrophysiology. Drugs R D. 2003; 4(2): 83-89, indexed in Pubmed: 12718562.

17. Lie RH, Hasenkam JM, Nielsen TT, et al. Post-conditioning reduces infarct size in an open-chest porcine acute ischemiareperfusion model. Acta Anaesthesiol Scand. 2008; 52(9): 1188-1193, doi: 10.1111/j.1399-6576.2008.01756.x, indexed in Pubmed: 18823456.

18. Abraham WT, Adams KF, Fonarow GC, et al. In-hospital mortality in patients with acute decompensated heart failure requiring intravenous vasoactive medications: an analysis from the Acute Decompensated Heart Failure National Registry (ADHERE). J Am Coll Cardiol. 2005; 46(1): 57-64, doi: 10.1016/j. jacc.2005.03.051, indexed in Pubmed: 15992636.

19. Takahama H, Yokoyama H, Kada A, et al. Extent of heart rate reduction during hospitalization using beta-blockers, not the achieved heart rate itself at discharge, predicts the clinical outcome in pa- 
tients with acute heart failure syndromes. J Cardiol. 2013; 61(1): 58-64, doi: 10.1016/j.jjcc.2012.08.014, indexed in Pubmed: 23165149.

20. Morelli A, Ertmer C, Westphal M, et al. Effect of heart rate control with esmolol on hemodynamic and clinical outcomes in patients with septic shock: a randomized clinical trial. JAMA. 2013; 310(16): 1683-1691, doi: 10.1001/jama.2013.278477, indexed in Pubmed: 24108526.

21. Sargento L, Satendra M, Longo S, et al. Heart rate reduction with ivabradine in patients with acute decompensated systolic heart failure. Am J Cardiovasc Drugs. 2014; 14(3): 229-235, doi: 10.1007/s40256-013-0060-1, indexed in Pubmed: 24452599.

22. Cavusoglu Y, Mert U, Nadir A, et al. Ivabradine treatment prevents dobutamine-induced increase in heart rate in patients with acute decompensated heart failure. J Cardiovasc Med (Hagerstown). 2015; 16(9): 603-609, doi: 10.2459/ JCM.0000000000000033, indexed in Pubmed: 24922198.
23. Barillà F, Pannarale G, Torromeo C, et al. Ivabradine in patients with ST-elevation myocardial infarction complicated by cardiogenic shock: a preliminary randomized prospective study. Clin Drug Investig. 2016; 36(10): 849-856, doi: 10.1007/s40261-0160424-9, indexed in Pubmed: 27312076.

24. Nuding S, Schröder J, Presek P, et al. Reducing elevated heart rates in patients with multiple organ dysfunction syndrome with the if (funny channel current) inhibitor ivabradine. Shock. 2018; 49(4): 402-411, doi: 10.1097/SHK.0000000000000992, indexed in Pubmed: 28930912.

25. Bakkehaug JP, Naesheim T, Torgersen Engstad E, et al. Reversing dobutamine-induced tachycardia using ivabradine increases stroke volume with neutral effect on cardiac energetics in left ventricular post-ischaemia dysfunction. Acta Physiol (Oxf). 2016; 218(2): 78-88, doi: 10.1111/apha.12704, indexed in Pubmed: 27145482. 